\title{
Uso y selección de microhábitat en un ensamble de anuros del Chaco Serrano de Argentina
}

\author{
Rodrigo A. Nieva Cocilio ${ }^{1,2}$, Juan C. Acosta ${ }^{2} \&$ Graciela M. Blanco ${ }^{2}$ \\ 1. Consejo Nacional de Investigaciones Científicas y Técnicas, Argentina; nievarodrigo@gmail.com \\ 2. Gabinete Diversidad y Biología de Vertebrados del Árido. Departamento de Biología, FCEFN, Universidad Nacional \\ de San Juan, Avenida Ignacio de la Roza 590, Rivadavia J5400DCS, San Juan, Argentina; jcacostasanjuan@gmail.com, \\ blancogracielam@gmail.com
}

\author{
Recibido 17-I-2020. Corregido 17-IV-2020. Aceptado 01-VI-2020.
}

\begin{abstract}
Microhabitat use and selection by an anuran assemblage from the Chaco Serrano of Argentina. Introduction: Research on spatial resource usage and partition strategies is important to understand the mechanisms of coexistence in sympatric amphibian species, even more when there are temporal variations in habitat availability. Objective: To learn about the patterns of microhabitat use, its seasonal variations and the possible influence of phylogeny on an anuran assembly of the Chaco Serrano in Argentina. Methods: Samplings were carried out in the Valle Fértil Natural Park protected area, Valle Fértil Department, San Juan, between 2012 and 2017. In the field, we recorded the microhabitat where each specimen was found, and we also measured site variables. In addition, microhabitats availability was determined. Data were analyzed using Manly's selectivity index. Generalized linear models (GLM) were used to assess temporal variations in microhabitat use. Results: The species evaluated were: Rhinella arenarum arenarum, Leptodactylus latrans, Pleurodema tucumanum and Odontophrynus occidentalis. All species showed differences in usage frequencies: $R$. a. arenarum showed preferences for rocky and aquatic sites, $P$. tucumanum showed preferences towards bare soil and rocky sites, L. latrans and $O$. occidentalis showed greater preferences for rocky and aquatic sites. All species but Odontophrynus exhibited seasonal variations in microhabitat selection and usage. Odontophrynus occidentalis showed differences in usage proportions among microhabitats. Conclusions: This study shows plasticity in microhabitat usage as an important determinant of anuran spatial distribution, without apparent restrictions imposed by space competition or phylogeny. When species activity is coincident, the space resource may be distributed in a way that species overlap is reduced.
\end{abstract}

Key words: amphibians; resource partitioning; Rhinella; Pleurodema; Leptodactylus; Odontophrynus; plasticity.

Nieva Cocilio, R.A., Acosta, J.C., \& Blanco, G.M. (2020). Uso y selección de microhábitat en un ensamble de anuros del Chaco Serrano de Argentina. Revista de Biología Tropical, $68(3), 862-872$.

El conocimiento del uso del espacio por parte de las especies es importante ya que en éste están disponibles los recursos potencialmente utilizables por los organismos (Begon, Harper, \& Townsend, 1997). Desde el punto de vista ecológico, la selección de microhábitat puede influenciar los procesos fisiológicos, las dinámicas de poblaciones y las comunidades. Por este motivo, las estrategias de forrajeo, disponibilidad de alimento, rendimiento locomotor, evasión de los depredadores y la competencia pueden llevar a diferentes usos y requerimientos de hábitats (Toft, 1985; Smith \& Ballinger, 2001).

La distribución espacial y los patrones de uso del hábitat están influenciados por una multitud de factores, tanto recientes como pasados (de Oliveira \& Eterovick, 2010). Entre 
los factores recientes, la influencia de la complejidad del hábitat, las interacciones bióticas, las restricciones fisiológicas específicas y los parámetros abióticos (como las condiciones climáticas) limitan la aparición de especies a solo unos pocos hábitats $\mathrm{y}$ ecosistemas dentro de una amplia gama de tipos disponibles (Baber, Fleishman, Babbitt, \& Tarr, 2004; Parris, 2004). Entre los factores pasados, la historia evolutiva de cada linaje puede determinar preferencias o restricciones que son producto de interacciones ecológicas pasadas, como la depredación y la competencia, o deberse a factores históricos, como las tasas de especiación y eventos de aislamiento o conexión de biotas (Hecnar, Casper, Russell, Hecnar, \& Robinson, 2002; Zimmerman \& Simberloff, 1996).

La evaluación de las respuestas de anfibios a cambios en la disponibilidad de hábitat, especialmente cuando los que prefieren no se encuentran disponibles, provee un indicador importante de plasticidad fisiológica y en el comportamiento. Por ende, la necesidad de determinar la selección o evasión de un microhábitat en particular en relación con su disponibilidad ha sido reconocida como un primer paso hacia el entendimiento y la explicación de las interacciones ecológicas entre los organismos y su ambiente (Law \& Dickman, 1998). Es decir que, conocer la relación entre la abundancia de las especies y las características de los microhábitats permite a los ecólogos hacer predicciones acerca de las respuestas de las especies a cambios antropogénicos y naturales en su hábitat (Martin \& Salvador, 1995).

En general los patrones de uso diferencial de los recursos disponibles pueden favorecer la sintopía de especies en un ensamble, ya que reducen el solapamiento y posibilitan la coexistencia de un mayor número de especies (Afonso \& Eterovick, 2007). Particularmente en anfibios tanto el uso de hábitat como los patrones de distribución, diversidad y abundancia están fuertemente relacionados con las variaciones temporales y estacionales, ya que dependiendo la temporada del año la disponibilidad de los hábitats puede variar, así como la presencia y persistencia de las especies en estos (Toft, 1980; Wilbur, 1987).

Son escasos los trabajos sobre uso de microhábitat en ensambles de anuros de Argentina (Duré, Kehr, Schaefer, \& Marangoni, 2008; Stellatelli \& Vega, 2010). Por este motivo, los objetivos de este trabajo fueron: 1) describir patrones de uso y selección del microhábitat con relación a su disponibilidad, 2) analizar la existencia de variaciones estacionales en la preferencia de microhábitat y 3) evaluar la posible influencia de la filogenia en la selección y uso del microhábitat por parte de cada una de las especies del ensamble estudiadas.

\section{MATERIALES Y MÉTODOS}

Área de estudio: Los muestreos se realizaron en la localidad de La Majadita, Departamento Valle Fértil, Provincia de San Juan, Argentina. Este sector se encuentra dentro de un Área Protegida denominada Parque Natural Valle Fértil. Pertenece a la Ecorregión del Chaco Seco, Distrito Serrano, en la ladera oriental de la Sierra de Valle Fértil y de la Huerta $\left(30^{\circ} 70^{\prime}\right.$ S \& $\left.67^{\circ} 49^{\prime} \mathrm{W}\right)$. La temperatura media anual es de $19.5^{\circ} \mathrm{C}$. El mes más cálido es enero, con $26^{\circ} \mathrm{C}$, mientras que el más frío es julio, con $11{ }^{\circ} \mathrm{C}$. El régimen de precipitaciones es estival, con medias anuales que superan los $400 \mathrm{~mm}$. El área posee una amplia red de cursos de agua temporarios que en general bajan por las laderas de las sierras y cuyo régimen hídrico es de alimentación pluvial (Brown \& Pacheco, 2006; Márquez, 1999).

Trabajo de campo: Se realizaron muestreos mensuales durante tres periodos de actividad de las especies, desde septiembre hasta abril, entre el 2012 y 2017. Los muestreos de uso de microhábitat se llevaron a cabo mediante caminatas nocturnas a lo largo de 26 transectas en faja de $10 \mathrm{~m}$ de ancho por $50 \mathrm{~m}$ de largo, establecidas sobre el lecho del río, en las márgenes del mismo y en las zonas de inundación del área de estudio (Heyer, Donnelly, McDiarmid, Hayek, \& Foster, 2001). Cada anuro observado durante el recorrido de 
transectas fue identificado según su especie, registrando el sitio dónde se halló al momento del avistamiento con el fin de asignarlo a categorías de microhábitat previamente establecidas: a) agua, sitios con presencia de agua, corriente o estancada, profunda (mayor a 0.70 m) o superficial (menor a $0.15 \mathrm{~m}$ ); b) suelo desnudo, sitios con arena, barro o grava con ausencia de vegetación; c) vegetación, sustratos terrestres con vegetación herbácea o arbustiva baja (siempre menor a $0.90 \mathrm{~m}$ ); d) roca, sitios rocosos formados por guijarros o rocas más grandes aisladas; e) vegetación acuática, todo tipo de vegetación en el agua y también aglomeraciones de algas sobre el agua. Para evaluar la disponibilidad de microhábitats se utilizaron las mismas transectas mencionadas anteriormente. Se dispusieron cuadrados de 10 x $10 \mathrm{~m}$ dentro de cada transecta, manteniendo siempre el margen derecho del lecho del río y separados uno de otro por $10 \mathrm{~m}$ de distancia (3 cuadrados por transecta en total). Dentro de cada cuadrado se midieron en forma de porcentaje los microhábitats presentes (disponibles). Para llevar a cabo los muestreos se contó con los permisos correspondientes otorgados por la Dirección de Conservación y Áreas Protegidas, Secretaría Ambiente y Desarrollo Sustentable, Subsecretaría de Conservación y Áreas Protegidas de la provincia de San Juan (Exp. no. 13004047, J.C.A.).

Para evaluar la selección de hábitat, se aplicó un diseño de uso-disponibilidad, según lo propuesto por Johnson, Nielsen, Merrill, McDonald, y Boyce (2006), debido a que la identificación precisa de puntos no utilizados puede ser poco práctica o imposible. Para obtener una función de selección de recursos se calculó el índice de Manly (Manly, McDonald, Thomas, McDonald, \& Erickson, 2002). Un índice de $1 / \mathrm{k}$ ( $\mathrm{k}$ = número de microhábitats) indica que no hay selección, un índice $>1 / \mathrm{k}$ indica selección, y un índice $<1 / \mathrm{k}$ indica evitación o rechazo. Para probar la confiabilidad de los índices de selección, se estimaron los intervalos de confianza del $95 \%$ del índice de selección por remuestreo (bootstrap), tomando una muestra aleatoria con reemplazo (500 veces) de sitios usados y disponibles en todos los microhábitats. El índice de selección fue significativamente diferente si el intervalo de confianza no contenía el valor $1 / \mathrm{k}$.

Para analizar las variaciones temporales en la selección de microhábitats se agruparon los datos de uso y disponibilidad según sus fechas en estaciones (Poblete \& Ruiz, 2006): primavera, verano y otoño. Se utilizaron modelos lineales generalizados (GLM). La variable respuesta (uso: 1, disponibilidad: 0) se ajustó a una distribución binomial, cuya función de enlace es logit (regresión logística). Se usó el siguiente modelo: glm (Uso Estación + Microhábitat + Estación * Microhábitat, family = binomial $($ link = "logit")). El ajuste de los modelos se evaluó mediante pruebas $\mathrm{X}^{2}$. Finalmente, se llevó a cabo un análisis de ordenamiento filogenético PCO para evaluar la influencia de la filogenia en el uso y selección del microhábitat. El análisis de datos se llevó a cabo mediante el software R (R Core Team, 2018).

\section{RESULTADOS}

Las especies del ensamble estudiadas, de acuerdo a su detección durante los periodos de muestreo, fueron: Rhinella arenarum arenarum (Bufonidae) (Hensel, 1867), común en ambientes naturales y antrópicos de monte y chaco. Tamaño máximo: $150 \mathrm{~mm}$. Su dieta es generalista, basada en artrópodos. Su reproducción comienza a fines de agosto, independientemente de las lluvias. En algunos lugares, donde las aguas poseen ciclos, la especie realiza dos oviposturas en cordones gelatinosos en el agua (Sanabria, Quiroga, \& Acosta, 2005). Leptodactylus latrans (Leptodactylidae) (Linnaeus, 1758), especie generalmente asociada a humedales. Tamaño máximo: $160 \mathrm{~mm}$. Su dieta es generalista, basada en artrópodos y vertebrados pequeños. Su actividad reproductiva sucede en primavera-verano. Los huevos son colocados en un nido de espuma realizado por la pareja sobre el agua y las hembras de la especie poseen cuidados parentales (Duellman \& Trueb, 1994; Rodríguez-Muñoz, 2016). Pleurodema tucumanum (Leptodactylidae) (Freiberg, 1942), 
especie cavícola y de ambientes arenosos de chaco. Tamaño máximo: $45 \mathrm{~mm}$. Su dieta es generalista, basada en artrópodos. Su reproducción se relaciona directamente con la temperatura ambiental, es decir primavera-verano. Deposita sus huevos en nidos de espuma sobre el agua (Rodriguez-Muñoz, Martínez, Acosta, \& Blanco, 2019). Odontophrynus occidentalis (Berg, 1896), especie cavícola. Los individuos pasan las épocas de sequía enterrados. Tamaño máximo: $65 \mathrm{~mm}$. Su dieta es generalista, basada en artrópodos. Su reproducción es estival. Deposita los huevos individualmente en el fondo lodoso de arroyos con vegetación acuática (Cei, 1980). Odontophrynus occidentalis estuvo presente en el ensamble sólo en el tercer periodo de muestreo. Por este motivo el número total de ejemplares registrados de esta especie resultó bajo y menor al del resto de las especies del ensamble estudiadas.
Índices de selección de microhábitat: Rhinella arenarum arenarum presentó mayores proporciones de uso versus disponibilidad hacia los microhábitats agua y roca (Fig. 1A). El índice de Manly corroboró esta tendencia, ya que indicó una selección importante hacia ambos ambientes (agua $=2.19$, roca $=4.31$ ). Además, el índice de selección reveló diferencias significativas para todos los microhábitats excepto para vegetación, ya que el intervalo de confianza incluyó el valor $1 / \mathrm{k}(1 / \mathrm{k}=0.2$, Fig. 2A), es decir que $R$. a. arenarum evitaría ocupar microhábitats con vegetación. Por el contrario, como ya se mencionó, esta especie prefiere o selecciona ambientes acuáticos (con o sin vegetación acuática) y rocosos.

Pleurodema tucumanum mostró una mayor proporción de uso hacia los microhábitats de suelo, respecto a la disponibilidad y respecto a las proporciones de uso de los demás
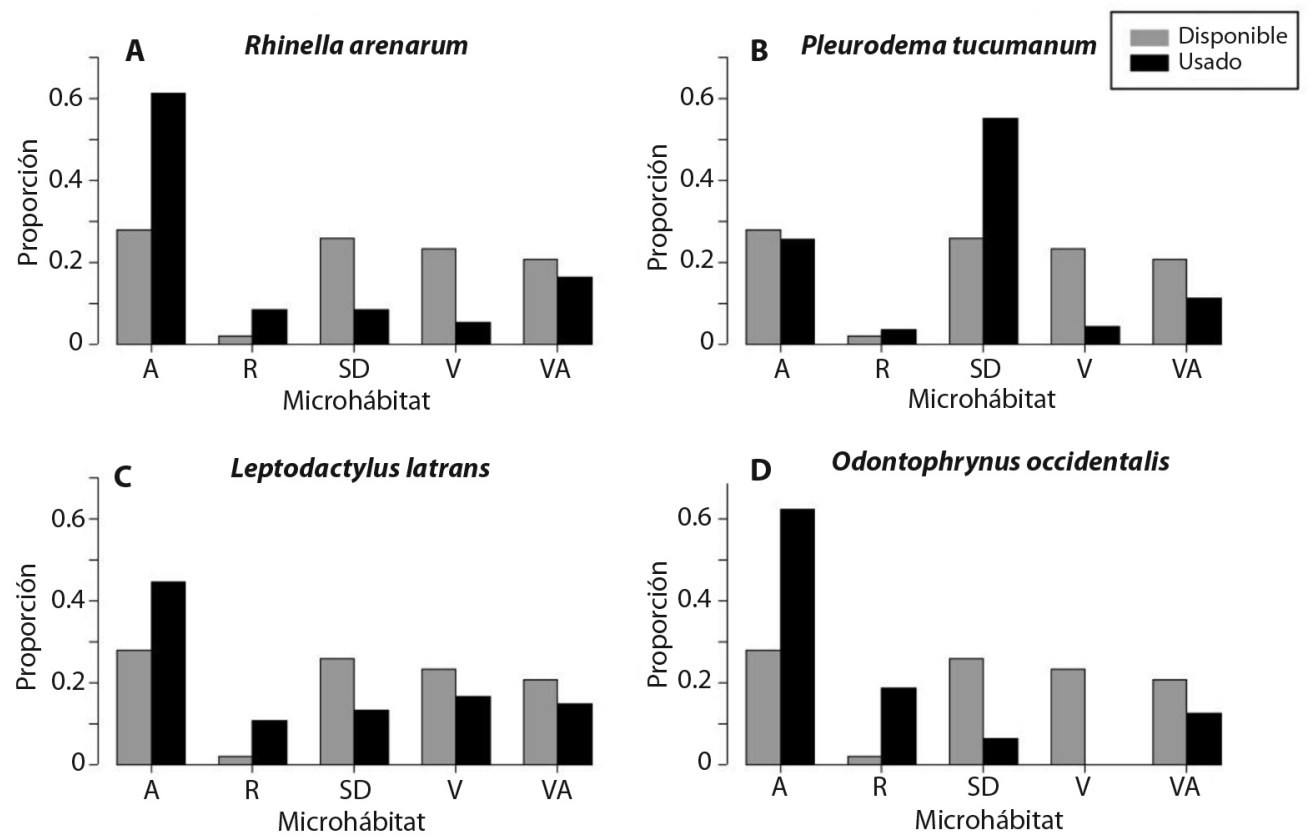

Fig. 1. Proporciones de uso y disponibilidad de cada tipo de microhábitat para cuatro especies de anuros: (A) Rhinella arenarum arenarum, (B) Pleurodema tucumanum, (C) Leptodactylus latrans y (D) Odontophrynus occidentalis. Microhábitats: $\mathrm{A}=$ agua, $\mathrm{R}=$ roca, $\mathrm{SD}=$ suelo desnudo, $\mathrm{V}=$ vegetación, $\mathrm{VA}=$ vegetación acuática.

Fig. 1. Usage (black) and availability (gray) proportions of each microhabitat type for four anuran species: (A) Rhinella arenarum arenarum, (B) Pleurodema tucumanum, (C) Leptodactylus latrans and (D) Odontophrynus occidentalis. Microhabitats: $\mathrm{A}=$ water, $\mathrm{R}=$ rock, $\mathrm{SD}=$ bare soil, $\mathrm{V}=$ vegetation, $\mathrm{VA}=$ aquatic vegetation. 

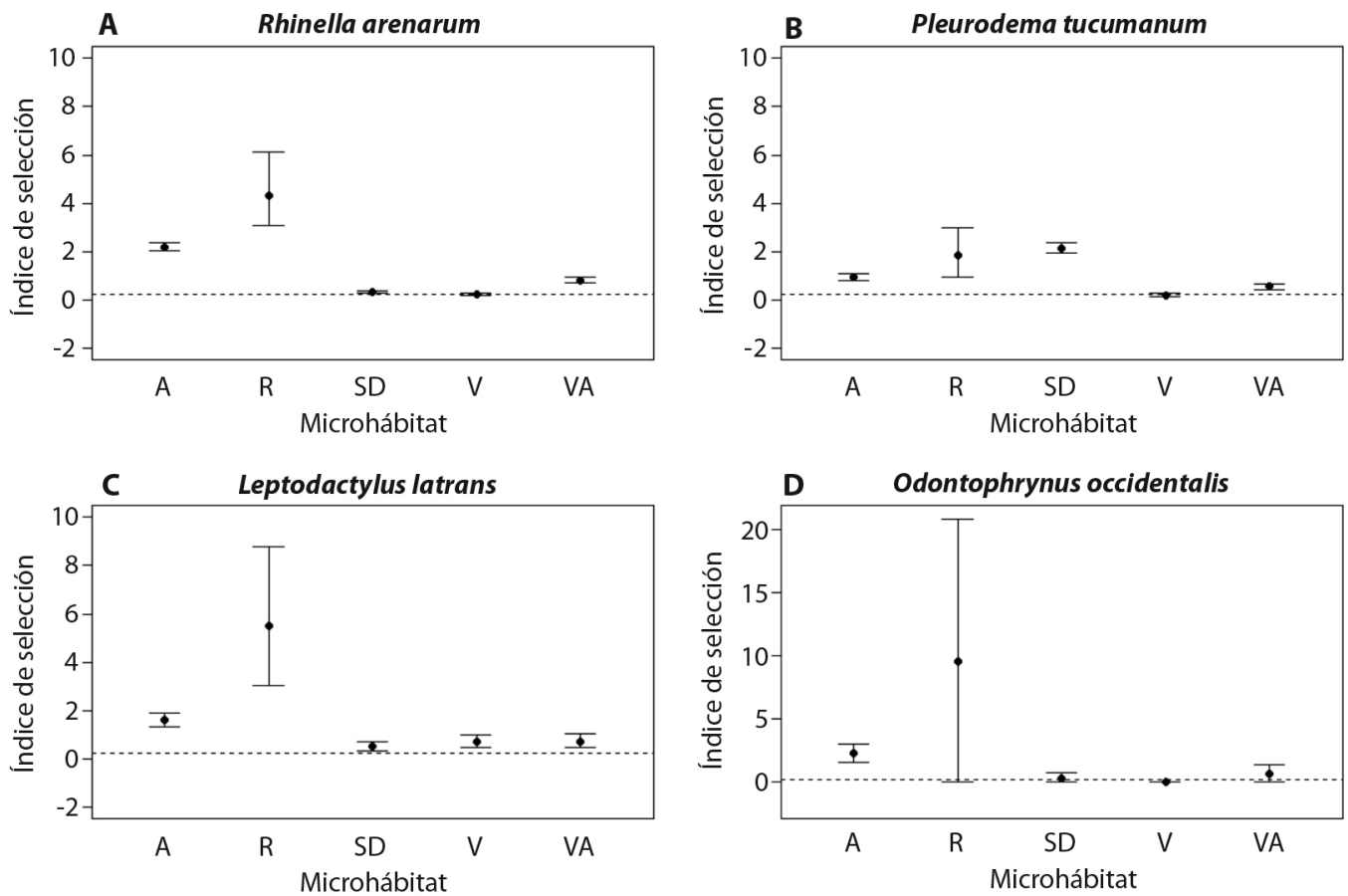

Fig. 2. Índices de selección (puntos) e intervalos de confianza del $95 \%$ (bigotes) de cada microhábitat en cuatro especies de anuros: (A) Rhinella arenarum arenarum, (B) Pleurodema tucumanum, (C) Leptodactylus latrans y (D) Odontophrynus occidentalis. Microhábitats: $\mathrm{A}=$ agua, $\mathrm{R}=$ roca, $\mathrm{SD}=$ suelo desnudo, $\mathrm{V}=$ vegetación, $\mathrm{VA}=$ vegetación acuática. $\mathrm{La}$ línea punteada indica el valor $1 / \mathrm{k}=0.2$.

Fig. 2. Selection indexes (points) and $95 \%$ confidence intervals (whiskers) of each microhabitat in four anuran species: (A) Rhinella arenarum arenarum, (B) Pleurodema tucumanum, (C) Leptodactylus latrans and (D) Odontophrynus occidentalis. Microhabitats: $\mathrm{A}$ = water, $\mathrm{R}=$ rock, $\mathrm{SD}=$ bare soil, $\mathrm{V}=$ vegetation, $\mathrm{VA}=$ aquatic vegetation. The dotted line indicates the $1 / \mathrm{k}$ value $=0.2$

microhábitats (Fig. 1B). Los índices de selección exhibieron una marcada preferencia de los microhábitats suelo (Manly $=2.12$ ) y roca $($ Manly $=1.85)($ Fig. 2B), mientras que el sitio vegetación presentó un índice de selección apenas inferior al valor $1 / \mathrm{k}$, es decir que esta especie evitaría ligeramente estos ambientes. Estas tendencias se corroboraron con los intervalos de confianza de los índices, que excluyeron el valor $1 / \mathrm{k}$ para todos los microhábitats excepto para vegetación (Fig. 2B).

Para Leptodactylus latrans las proporciones de uso respecto a disponibilidad fueron mayores para los microhábitats agua y roca, exhibiendo el primero una intensidad de uso mayor al resto de los sitios (Fig. 1C). No obstante, el índice de selección fue mayor y de alto valor para roca (Manly $=5.49)$, es decir que la probabilidad de selección de este microhábitat por parte de la especie es mayor respecto a la probabilidad de selección de los demás sitios. Los intervalos de confianza fueron significativos para todos los sitios, demostrando que esta especie selecciona una amplia variedad de microhábitats y no muestra rechazo o evasión hacia ninguno de ellos (Fig. 2C).

Odontophrynus occidentalis exhibió una alta proporción de uso de agua y en menor medida del ambiente rocoso (roca) respecto a la disponibilidad de microhábitats (Fig. 1D). La proporción de uso de vegetación fue nula y tampoco se pudo llevar a cabo el cálculo del índice de selección para este tipo de microhábitat (la especie no presenta selección) (Manly $=0$ ). El ambiente rocoso registró un alto índice de selección (Manly = 9.58), sin embargo, 
el intervalo de confianza incluyó el valor $1 / \mathrm{k}=$ 0.2 (Fig. 2D). Estos resultados pueden deberse al bajo número de usos registrados frente a un alto número de sitios disponibles. Por otro lado, el sitio agua presentó un índice de selección positiva significativo (Manly $=2.23$ ), siendo el único sitio cuyo intervalo de confianza no contuvo el valor 1 / k (Fig. 2D).

Variaciones temporales en la selección de microhábitat y filogenia: Para todas las especies, el modelo lineal generalizado (GLM) seleccionado con base en el criterio de Akaike (AIC) incluyó los efectos de las variables explicativas (estación y microhábitat) por separado y los efectos de la interacción entre ambas variables. En el GLM propuesto para $R$. $a$. arenarum, tanto la variable explicativa estación $\left(\mathrm{X}^{2}=66, \mathrm{gl}=2, \mathrm{P}<0.001\right)$, la variable microhábitat $\left(X^{2}=510.89, \mathrm{gl}=4, \mathrm{P}<0.001\right)$, como la interacción de microhábitat * estación tuvieron efectos significativos $\left(\mathrm{X}^{2}=44.23, \mathrm{gl}=8, \mathrm{P}<\right.$ 0.001 ) en la variable respuesta (sitios usados o disponibles). Esto quiere decir que $R$. a. arenarum presenta variaciones en el uso de hábitat entre estaciones y entre microhábitats. Según los estimados del modelo los microhábitats que presentaron mayores diferencias significativas de uso entre estaciones fueron roca en otoño y vegetación acuática en primavera. Por otro lado, el microhábitat agua fue el que presentó mayores diferencias entre microhábitats, con una proporción de uso mucho mayor al resto (Fig. 3A).

En el GLM de P. tucumanum las dos variables explicativas y su interacción presentaron efectos significativos (estación: $\mathrm{X}^{2}=884.17$, $\mathrm{gl}=2, \mathrm{P}<0.001$; microhábitat: $\mathrm{X}^{2}=170.26, \mathrm{gl}=$ 4, $\mathrm{P}<0.001$; interacción: $\mathrm{X}^{2}=70.05, \mathrm{gl}=8, \mathrm{P}$ $<0.001)$ en la variable respuesta. Por ende, $P$. tucumanum varía el uso y selección de los distintos microhábitats a través de las estaciones.
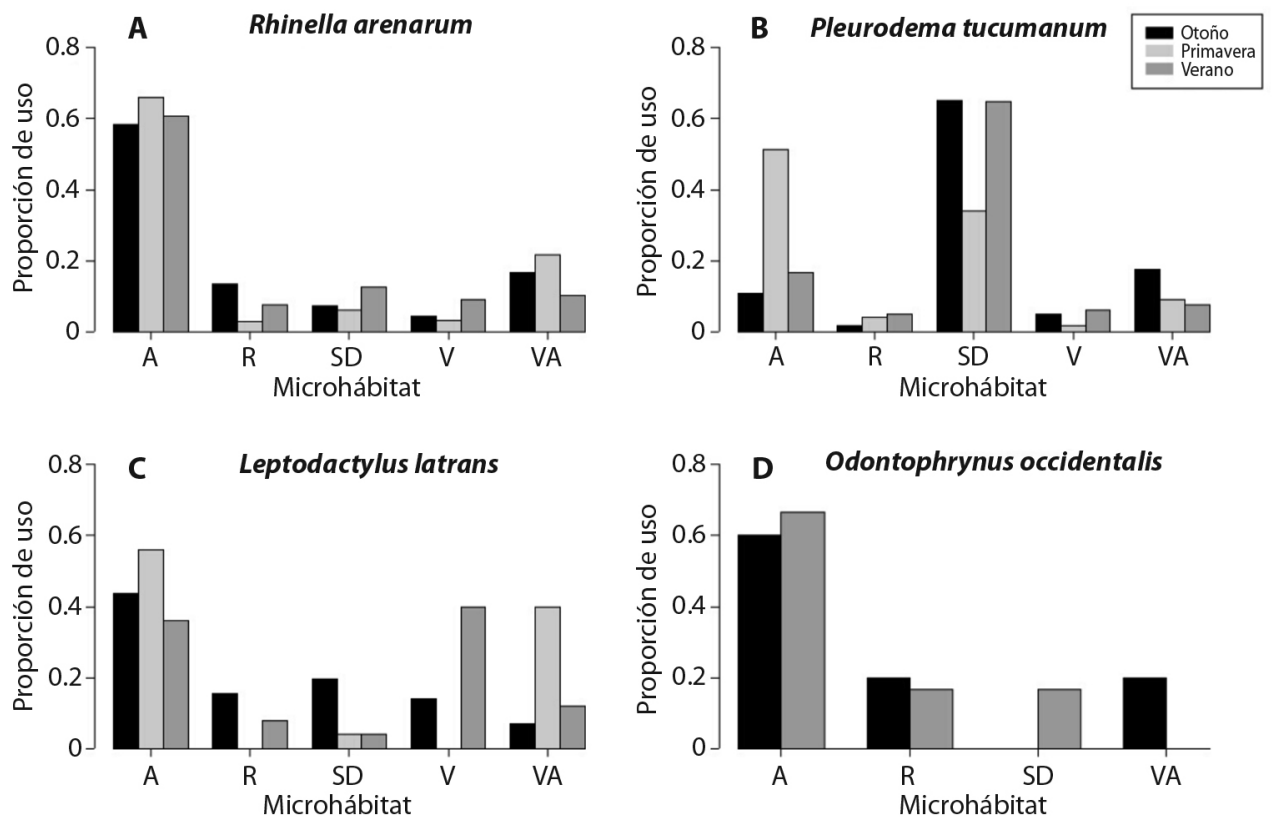

Fig. 3. Proporciones de uso de cada tipo de microhábitat para cuatro especies de anuros: (A) Rhinella arenarum arenarum, (B) Pleurodema tucumanum, (C) Leptodactylus latrans y (D) Odontophrynus occidentalis, en tres estaciones. Microhábitats: $\mathrm{A}=$ agua, $\mathrm{R}=$ roca, $\mathrm{SD}=$ suelo desnudo, $\mathrm{V}=$ vegetación, $\mathrm{VA}=$ vegetación acuática.

Fig 3. Usage proportions of each microhabitat type for four anuran species: (A) Rhinella arenarum arenarum, (B) Pleurodema tucumanum, (C) Leptodactylus latrans and (D) Odontophrynus occidentalis, in three seasons. Microhabitats: $\mathrm{A}=$ water, $\mathrm{R}=$ rock, $\mathrm{SD}=$ bare soil, $\mathrm{V}=$ vegetation, $\mathrm{VA}=$ aquatic vegetation. Seasons: Otoño = fall, Primavera $=$ spring, Verano $=$ summer. 
Los microhábitats que presentaron mayores diferencias significativas fueron agua, suelo y vegetación acuática (Fig. 3B), observándose, por un lado, mayor proporción de uso de cuerpos de agua en primavera y por otro, altas proporciones de uso del microhábitat suelo en verano y otoño.

El GLM aplicado para L. latrans resultó con efectos significativos de la variable explicativa estación $\left(X^{2}=52.42, \mathrm{gl}=2, \mathrm{P}<0.001\right)$, la variable microhábitat $\left(\mathrm{X}^{2}=34.50, \mathrm{gl}=4, \mathrm{P}<\right.$ 0.001) y también la interacción de microhábitat * estación $\left(\mathrm{X}^{2}=35.65, \mathrm{gl}=8, \mathrm{P}<0.001\right)$ sobre la variable respuesta. Por lo tanto, L. latrans realiza un uso diferencial de los microhábitats a través de las temporadas. En la Fig. 3C puede observarse, según los estimados del modelo, que todos los microhábitats presentaron diferencias significativas de uso respecto a las estaciones, destacándose un marcado uso de vegetación acuática y agua en primavera; preferencia que cambia hacia el verano, cuando utilizan la vegetación en mayor proporción.

Para $O$. occidentalis las diferencias estacionales en la selección de microhábitat se evaluaron sólo para el verano y otoño, ya que en primavera no se hallaron individuos en las transectas y por consiguiente no se registraron observaciones de uso. El GLM aplicado para esta especie arrojó efectos significativos sobre la variable respuesta solamente por parte de la variable explicativa microhábitat $\left(X^{2}=22.36\right.$, $\mathrm{gl}=4, \mathrm{P}<0.001$ ), pues no hubo efecto significativo de la estación $\left(\mathrm{X}^{2}=3.12, \mathrm{gl}=1, \mathrm{P}=0.08\right)$, ni en la interacción de microhábitat * estación $\left(\mathrm{X}^{2}=4.68, \mathrm{gl}=8, \mathrm{P}=0.32\right)$. Estos resultados indican que para $O$. occidentalis no varían las proporciones de uso entre las estaciones analizadas (verano y otoño), pero si varían las proporciones de uso entre los distintos microhábitats, como se mencionó anteriormente en resultados de índices de selección, siendo el microhábitat agua el de mayor frecuencia de uso tanto en verano como en otoño (Fig. 3D).

En la Fig. 4 se puede observar el árbol de especies utilizado para llevar a cabo el análisis filogenético de acuerdo a la filogenia de Montero y Autino (2018). El análisis CPO resultó

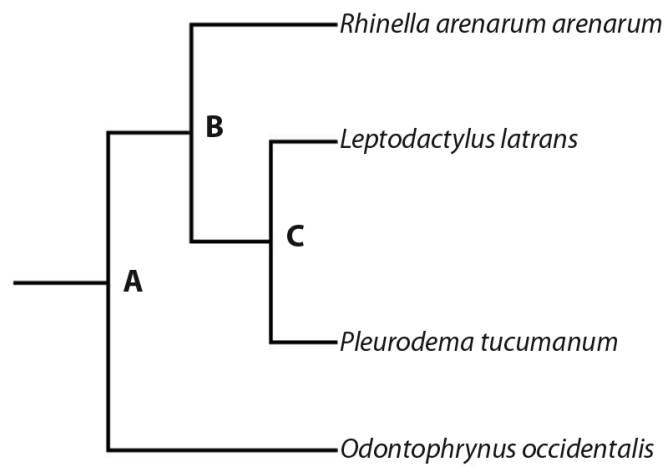

Fig. 4. Grupos individuales utilizados en el análisis de ordenamiento filogenético para uso de microhábitat. Filogenia basada en Montero y Autino (2018).

Fig. 4. Individual groups used in the phylogenetic ordination analysis for microhabitat use. Phylogeny based on Montero and Autino (2018).

no significativo para los tres nodos estudiados. Las permutaciones de Monte Carlo (basadas en 9999 permutaciones) de la ordenación canónica no revelaron ningún efecto filogenético significativo en los microhábitats utilizados o del ensamble de anuros (CCA: $\mathrm{F}=1.37, \mathrm{P}=0.37$ ).

\section{DISCUSIÓN}

Este trabajo constituye el primer estudio de uso de microhábitat para un ensamble de anuros del Chaco de San Juan, Argentina. Kacoliris, Williams, Quiroga, Molinari, y Vicente (2011) propusieron que las principales causas asociadas al uso diferencial de hábitats/ microhábitats tienen relación con la termorregulación, alimentación, reproducción y detección de depredadores. El hecho de presentarse índices positivos de selección hacia ambientes acuáticos podría vincularse con estos aspectos mencionados y estrechamente relacionados en los anfibios (Tracy, 1976). El agua posee mayor inercia térmica que el suelo y en consecuencia podría ser un microhábitat adecuado para la termorregulación de las especies de anuros nocturnas, sobre todo cuando la temperatura ambiental es baja y/o el viento es fuerte. Navas (1996, 2003), propone que la altitud, la hora de actividad y el uso de microhábitat influyen en la temperatura corporal de los anfibios. Así es 
que, la termorregulación conductual es difícil de separar de los mecanismos de comportamiento para la conservación del agua, ya que cuando la temperatura aumenta o la humedad disminuye, la tasa de pérdida de agua aumenta debido a la permeabilidad de la piel (Vitt \& Caldwell, 2009).

Los resultados hallados coinciden con los obtenidos por Ponssa (2004) en una comunidad de anuros de Panamá; Vargas y Castro (1999) y Zorro-Ceron (2007) para grupos de anuros de Colombia; Almeida-Gomes, Hatano, Van Sluys, y Rocha (2007) para dos especies de Brasil; Wachlevski, De Souza, Kopp, y Eterovick (2008) para Crossodactylus en Brasil y Pereyra, Lescano, y Leynaud (2011) para Melanophryniscus de Argentina. A pesar de tratarse de ensambles y especies distintas a las de este estudio, demuestran preferencias de microhábitats similares. Contrariamente, se han reportado resultados indicando que las especies de un ensamble pueden segregar el microhábitat utilizando diferentes alturas o estratos verticales (Alfonso \& Eterovick, 2007; Muñoz-Guerrero, Serrano, \& Ramírez-Pinilla, 2007), hecho que no ocurrió en este trabajo debido, por ejemplo, a la ausencia de la familia Hylidae en las especies del ensamble.

Pleurodema tucumanum ocupa cuerpos de agua de manera oportunista para reproducirse, pero refleja preferencia específica por sitios con suelo desnudo, en los cuales podría llevar a cabo el resto de sus actividades (termorregulación y alimentación principalmente). Teniendo en cuenta que $P$. tucumanum es la especie más pequeña del ensamble, Gillespie, Lockie, Scroggie, \& Iskandar (2004) sugieren que el tamaño y la morfología de las especies son factores potencialmente importantes en la partición de recursos dentro de las comunidades de anfibios. Simon y Toft (1991) proponen que los estratos bajos y sustratos como hojarasca y suelo desnudo proveen a las especies pequeñas de anuros terrestres gran cantidad de recursos para forrajeo. Esto podría explicar el "rechazo" de esta especie hacia sustratos con vegetación, dando lugar a índices de selección levemente negativos. Además, la selección diferencial de $P$. tucumanum, concuerda con el estudio de Muñoz-Guerrero et al. (2007), encontrando que el tamaño corporal tuvo relación con el uso del microhábitat y que las especies de tamaño corporal pequeño ocuparon microhábitats diferentes a los de especies más grandes.

Los resultados hallados para Leptodactylus latrans concuerdan, en parte, con un estudio de Duré y Kehr (2004) para dos especies de Leptodactylus del nordeste argentino que mostraron preferencias por distintos tipos de microhábitats. Sin embargo, observaron a L. latinasus con mayor frecuencia en áreas con barro, suelo agrietado y pasto con barro; y a L. bufonius en zonas con tierra seca y pasto corto, contrariamente a los resultados de este estudio, en el cual $L$. latrans registró mayores proporciones de uso de ambientes acuáticos.

En el caso de Odontophrynus occidentalis, se debe ser cauto al realizar inferencias en cuanto a su selección o preferencia, ya que el bajo número de observaciones (uso) podría llevar a errores de interpretación de los índices de selección al comparar con el alto número de sitios disponibles. Interpretar la falta de preferencia de esta especie (índice igual a cero) como que el animal no está respondiendo hacia el microhábitat o que el microhábitat no es importante sería un error. Un coeficiente nulo de selección podría indicar un hábitat en el cual se analizó la selección en niveles distintos a los de disponibilidad, cómo es el caso de este trabajo, en lugar de un hábitat al que el animal no responde en absoluto (Beyer et al., 2010).

El hecho que se registraran mayores proporciones de uso del lecho del río y cuerpos de agua en primavera puede asociarse directamente con la actividad reproductiva del ensamble en esta estación. Gillespie et al. (2004) considera que la partición espacial y/o temporal de los recursos reproductivos es un mecanismo importante que permite a las especies sintópicas evitar la competencia y asegurar el éxito reproductivo, sobre todo en ambientes subtropicales donde hay restricciones abióticas en la disponibilidad temporal de hábitats para reproducción. Además, las preferencias de hábitats acuáticos pueden explicarse por los modos 
reproductivos de las especies del ensamble, consistentes en depositar un gran número de huevos en el agua, en cordones gelatinosos o nidos de espuma, donde eclosionan y se desarrollan los renacuajos (Duellman \& Trueb, 1994; Rodriguez-Muñoz et al., 2019).

Los sitios de canto y oviposición pueden señalarse como los principales recursos ambientales utilizados por anuros adultos, determinando los patrones de uso del hábitat y la distribución espacial de las especies sintópicas (de Oliveira \& Eterovick, 2010). Además, Leptodactylus latrans, presentó una proporción considerable de uso de vegetación acuática en primavera (Fig. 3C), resultado que concuerda con su modo reproductivo, consistente en la colocación de los huevos en nidos de espuma anclados a la vegetación.

Según los resultados del $\mathrm{CPO}$ el efecto histórico o filogenético no tendría influencia en la selección de microhábitat por parte de las especies estudiadas del ensamble, por el contrario, lo que determinaría la selección serían factores extrínsecos o ambientales. Una posible explicación a este hecho sería que los conjuntos de datos ecológicos de ensambles pobres en especies pueden sufrir deficiencias de muestreo de taxones, de modo que los efectos históricos reales sean indetectables porque varios taxones principales estén subrepresentados (Mesquita, Colli, França, \& Vitt, 2006). Además, la falta de especies estrechamente relacionadas también puede dificultar la detección del papel de la filogenia en las interacciones biológicas. Por otro lado, Eterovick et al. (2010) plantean que el uso del microhábitat no es un buen indicador filogenético para anfibios, a pesar de que encontraron algunas relaciones débiles en su trabajo.

Este estudio muestra la variabilidad en las adaptaciones de las especies y la plasticidad del uso de microhábitats como determinantes importantes de la distribución espacial de anuros, sin restricciones aparentes impuestas por la competencia por el espacio ni por la filogenia (de Oliveira \& Eterovick, 2010). Es decir, que cuando la actividad de cada una de las especies del ensamble es coincidente, el recurso espacial estaría siendo distribuido de tal manera que el solapamiento entre las especies sea disminuido.

Declaración de ética: los autores declaran que todos están de acuerdo con esta publicación y que han hecho aportes que justifican su autoría; que no hay conflicto de interés de ningún tipo; y que han cumplido con todos los requisitos y procedimientos éticos y legales pertinentes. Todas las fuentes de financiamiento se detallan plena y claramente en la sección de agradecimientos. El respectivo documento legal firmado se encuentra en los archivos de la revista.

\section{AGRADECIMIENTOS}

A Tomás Martínez, Melina Rodriguez, Rodrígo Gómez, Ana Galdeano, Lia Piaggio, Susana Castro, Gustavo Fava por su colaboración en los trabajos de campo. A la Dirección de Conservación, Subsecretaría de Conservación y Áreas Protegidas dependiente de la Secretearía de Ambiente y Desarrollo Sustentable de la provincia de San Juan por los permisos de colecta otorgados (No 58-2000-SA).

\section{RESUMEN}

Introducción: El estudio de las estrategias de uso y partición del recurso espacial resulta importante para entender los mecanismos de coexistencia de especies de anfibios simpátricas, más aún cuando existen variaciones temporales en la disponibilidad de hábitats. Objetivo: Conocer los patrones de uso de microhábitat, sus variaciones estacionales y la posible influencia de la filogenia en un ensamble de anuros del Chaco Serrano de Argentina. Métodos: Los muestreos se realizaron en el área protegida Parque Natural Valle Fértil, Departamento Valle Fértil, San Juan, entre el 2012 y 2017. En campo, se registró el microhábitat donde se encontró cada ejemplar y se midieron variables del sitio. Además, se determinó la disponibilidad de microhábitats. Los datos se analizaron mediante el índice de selectividad de Manly. Para evaluar variaciones temporales en el uso de microhábitats se utilizaron modelos lineales generalizados (GLM). Resultados: Las especies evaluadas fueron: Rhinella arenarum arenarum, Leptodactylus latrans, Pleurodema tucumanum y Odontophrynus occidentalis. Todas las especies mostraron diferencias en las frecuencias de uso: R. a. arenarum mostró preferencias hacia los sitios rocosos y acuáticos, $P$. tucumanum exhibió preferencias hacia los 
sitios con suelo desnudo y rocas, L. latrans y $O$. occidentalis presentaron mayores preferencias hacia los sitios rocosos y acuáticos. Todas las especies excepto Odontophrynus exhibieron variaciones estacionales en la selección y uso de los microhábitats. Odontophrynus occidentalis presentó diferencias en las proporciones de uso entre microhábitats. Conclusiones: Este estudio muestra la plasticidad en el uso de microhábitats como un determinante importante en la distribución espacial de anuros, sin restricciones aparentes impuestas por la competencia, el espacio ni la filogenia. Cuando la actividad de las especies es coincidente, el recurso espacial puede distribuirse de manera que se reduzca el solapamiento entre especies.

Palabras clave: anfibios; partición de recursos; Rhinella; Pleurodema; Leptodactylus; Odontophrynus; plasticidad.

\section{REFERENCIAS}

Afonso, L.G., \& Eterovick, P.C. (2007). Microhabitat choice and differential use by anurans in forest streams in southeastern Brazil. Journal of Natural History, 41(13-16), 937-948.

Almeida-Gomes, M., Hatano, F.H., van Sluys, M., \& Rocha, C.F.D. (2007). Diet and microhabitat use by two Hylodinae species (Anura, Cycloramphidae) living in sympatry and syntopy in a Brazilian Atlantic rainforest area. Iheringia Série Zoologia, 97, 27-30.

Baber, J.M., Fleishman, E., Babbitt, K.J., \& Tarr, T.L. (2004). The relationship between wetland hydroperiod and nestedness patterns in assemblages of larval amphibians and predatory macroinvertebrates. Oikos, 107(1), 16-27.

Begon, M., Harper, L.J., \& Townsend, C.R. (1997). Ecología. Individuos, poblaciones y comunidades. Barcelona, España: Ediciones Omega, S.A.

Berg, C. (1896). Batracios Argentinos. Enumeración sistemática, sinonímica y bibliográfica de los batracios de la República Argentina. Annales Museo Nacional de Historia Natural, 5(2), 147-226.

Beyer, H.L., Haydon, D.T., Morales, J.M., Frair, J.L., Hebblewhite, M., Mitchell, M., \& Matthiopoulos, J. (2010). The interpretation of habitat preference metrics under use-availability designs. Philosophical Transactions of the Royal Society of London B: Biological Sciences, 365(1550), 2245-2254.

Brown, A.D., \& Pacheco, S. (2006). Propuesta de actualización del mapa ecorregional de la Argentina. En A.D. Brown, U. Martínez Ortíz, M. Acerbi, \& J. Corcuera. La situación ambiental argentina 2005 (pp. 28-31). Argentina: Fundación Vida Silvestre Argentina.

Cei, J.M. (1980). Amphibians of Argentina (Vol. 2). Monitore Zoologico Italiano. Nuova Serie, Monographia, 2, 1-609. de Oliveira, F.F.R., \& Eterovick, P.C. (2010). Patterns of spatial distribution and microhabitat use by syntopic anuran species along permanent lotic ecosystems in the Cerrado of southeastern Brazil. Herpetologica, 66(2), 159-171.

Duellman, W.E. \& Trueb, L. (1994). Biology of Amphibians. Londres: John Hopkins University Press Ltd. London.

Duré, M.I., \& Kehr, A.I. (2004). Influence of microhabitat on the trophic ecology of two leptodactylids from northeastern Argentina. Herpetologica, 60(3), 295-303.

Duré, M.I., Kehr, A.I., Schaefer, E.F., \& Marangoni, F. (2008). Diversity of amphibians in rice fields from northeastern Argentina. Interciencia, 33(7), 528-531.

Eterovick, P.C., Rievers, C.R., Kopp, K., Wachlevski, M., Franco, B.P., Dias, C.J., Barata, I.M., Ferreira, A.D.M., \& Afonso, L.G. (2010). Lack of phylogenetic signal in the variation in anuran microhabitat use in southeastern Brazil. Evolutionary Ecology, 24(1), 1-24.

Freiberg, M.A. (1942). Enumeración sistemática y distribución geográfica de los batracios argentinos. Physis, 19, 219-240.

Gillespie, G.R., Lockie, D., Scroggie, M.P., \& Iskandar, D.T. (2004). Habitat use by stream-breeding frogs in south-east Sulawesi, with some preliminary observations on community organization. Journal of Tropical Ecology, 20, 439-448.

Hecnar, S.J., Casper, G.S., Russell, R.W., Hecnar, D.R., \& Robinson, J.N. (2002). Nested species assemblages of amphibians and reptiles in the Laurentian Great Lakes. Journal of Biogeography, 29, 475-489.

Hensel, R. (1867). Beiträge zur kenntniss der Wirbelthiere Südbrasiliens. Archiv für Naturgeschichte, 33(1), 120-162.

Heyer, W.R., Donnelly, M.A., McDiarmid, R.W., Hayek, L.C., \& Foster, M.S. (2001). Medición y Monitoreo de la Diversidad Biológica. Métodos Estandarizados Para Anfibios. Argentina: Editorial Universidad de La Patagonia.

Johnson, C.J., Nielsen, S.E., Merrill, E.H., McDonald, T.L., \& Boyce, M.S. (2006). Resource selection functions based on use-availability data: theoretical motivation and evaluation methods. Journal of Wildlife Manage, 70, 347-357.

Kacoliris, F.P., Williams, J.D., Quiroga, S., Molinari, A., \& Vicente, N. (2011). Ampliación del conocimiento sobre uso de hábitat en Liolaemus multimaculatus, sitios de fuga. Cuadernos de Herpetología, 25(1), 5-10.

Law, B.S. \& Dickman, C.R. (1998). The use of habitat mosaic by terrestrial vertebrate fauna: implications for conservation and management. Biodiversity Conservation, 7, 323-333. 
Linnaeus, C. (1758). Systema naturae per regna tria naturae, secundum classes, ordines, genera, species, cum characteribus, differentiis, synonymis, locis (Tomus I). Salvius. Holmiae: Editio decima, reformata.

Manly, B.F.J., McDonald, L.L., Thomas, D.L., McDonald, T.L., \& Erickson, E.P. (2002). Resource selection by animals: statistical design and analysis of field studies (2a Ed.). The Netherlands: Springer Netherlands.

Márquez, J. (1999). Las Áreas Protegidas de la Provincia de San Juan. Multequina, 8, 1-10.

Martin, J., \& Salvador, A. (1995). Microhabitat selection by the Iberian rock lizard Lacerta monticola: Effects on density and spatial distribution of individuals. Biological Conservation 79, 303-307.

Mesquita, D.O., Colli, G.R., França, F.G., \& Vitt, L.J. (2006). Ecology of a Cerrado lizard assemblage in the Jalapão region of Brazil. Copeia, 3, 460-471.

Montero, R., \& Autino, A.G. 2018. Sistemática y filogenia de los vertebrados, con énfasis en la fauna argentina (3a Ed.). San Miguel de Tucumán, Argentina: Editorial independiente.

Muñoz-Guerrero, J., Serrano, V.H., \& Ramírez-Pinilla, M.P. (2007). Uso de Microhábitat, Dieta y Tiempo de Actividad en Cuatro Especies Simpátricas de Ranas Hílidas Neotropicales (Anura: Hylidae). Caldasia, 29(2), 413-425.

Navas, C. (1996). The effect of temperature on the vocal activity of tropical anurans: a comparison of high and low-elevation species. Journal of Herpetology, $30,488-497$

Navas, C. (2003). Herpetological diversity along Andean elevational gradients: links with physiological ecology and evolutionary physiology. Comparative Biochemistry and Physiology Part A 133, 469-485.

Parris, K.M. (2004). Environmental and spatial variables influence the composition of frog assemblages in subtropical eastern Australia. Ecography, 27, 392-400.

Pereyra, L.C., Lescano, J.N., \& Leynaud, G.C. (2011). Breeding-site selection by red-belly toads, Melanophryniscus stelzneri (Anura: Bufonidae), in Sierras of Córdoba, Argentina. Amphibia-Reptilia, 32, 105-112.

Poblete, G.A., \& Ruiz, S.B. (2006). Las estaciones térmicas en el Valle de Tulum. UNSJ. FCEFN. Revista Ciencias, 1, 34-42.

Ponssa, M.L. (2004). Utilización espacial y temporal de una comunidad de anuros de Kent's Marsh (Gamboa, Panamá). Revista Española de Herpetología, 18, 5-18.

R Core Team. (2018). R: A language and environment for statistical computing. R Foundation for Statistical Computing, Vienna, Austria. Recuperado de http:// www.R-project.org/
Rodriguez Muñoz, M.J., Martínez, T.A., Acosta, J.C., \& Blanco, G.M. (2019). Foam nest construction and first report of agonistic behaviour in Pleurodema tucumanum (Anura: Leptodactylidae). Neotropical Biology and Conservation, 14(1), 117-128.

Sanabria, E., Quiroga, L., \& Acosta, J.C. (2005). Patrones de actividad temporal, estacional y uso de microhábitat de una población de adultos de Bufo arenarum, en los humedales de Zonda, San Juan, Argentina. Boletín de la Sociedad Herpetológica Mexicana, 13(2), 61-65.

Simon, M.P., \& Toft, C.A. (1991). Diet specialization in small vertebrates: Mite eating in Frogs. Oikos, 61, 263-278.

Smith, G., \& Ballinger, R. (2001). The Ecological Consequences of Habitat and Microhabitat Use in Lizards: a review. Contemporary Herpetology, 3, 1-37.

Stellatelli, O.A., \& Vega, L.E. (2010). Estructura del ensamble de anuros de la Reserva Integral Laguna de los Padres (Buenos Aires, Argentina). Cuadernos de Herpetología, 24(2), 111-122.

Toft, C. (1980). Feeding ecology of thirteen syntopic species of anurans in a seasonal tropical environment. Oecologia, 45, 131-141.

Toft, C.A. (1985). Resource partitioning in amphibians and reptiles. Copeia, 1, 1-21.

Tracy, C.R. (1976). Model of dynamic exchanges of water and energy between a terrestrial amphibian and its environment. Ecological Monographs, 46, 293-326.

Vargas, F., \& Castro, F.H. (1999). Distribución y preferencias de microhabitat en anuros (Amphibia) en bosque maduro y áreas perturbadas en Anchicayá, Pacífico Colombiano. Caldasia, 21, 95-109.

Vitt, L.J., \& Caldwell, J.P. (2009). Herpetology, An Introductory Biology of Amphibians \& Reptiles (3a Ed.). Academic Press.

Wachlevski, M., De Souza, P.H., Kopp, K., \& Eterovick, P.C. (2008). Microhabitat use and feeding habits of Crossodactylus bokermanni Caramaschi and Sazima, (Anura, Hylodidae) at a site in south-eastern Brazil. Journal of Natural History, 42(19-20), 1421-1434.

Wilbur, H.M. (1987). Regulation of structure in complex systems: experimental temporary pond communities. Ecology, 68(5), 1437-1452.

Zimmerman, B.L., \& Simberloff, D. (1996). An historical interpretation of habitat use by frogs in a central Amazonian forest. Journal of Biogeography, 23, 27-46

Zorro Ceron, J.P. (2007). Anuros de piedemonte llanero: diversidad y preferencias de microhábitat (Trabajo final de Licenciatura). Pontífica Universidad Javierana, Colombia. 